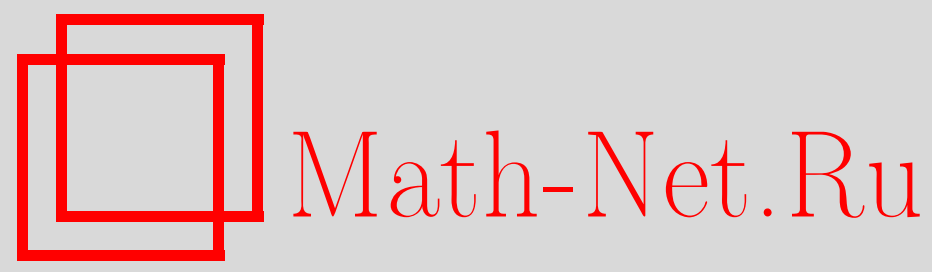

А. Ф. Васильев, Одна задача теории формаций конечных групп, Матем. заметки, 1997, том 62, выпуск 1, 52-58

DOI: https://doi.org/10.4213/mzm1586

Использование Общероссийского математического портала Math-Net.Ru подразумевает, что вы прочитали и согласны с пользовательским соглашением http://www. mathnet.ru/rus/agreement

Параметры загрузки:

IP : 54.197 .130 .99

26 апреля 2023 г., 13:11:42

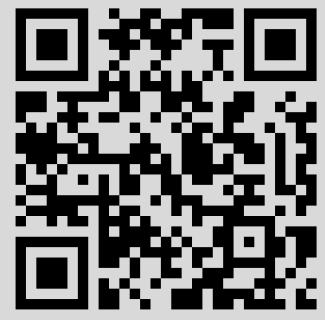




\title{
ОДНА ЗАДАЧА ТЕОРИИ ФОРМАЦИЙ КОНЕЧНЫХ ГРУПП
}

\author{
А. Ф. Васильев
}

В 1968 году Картером, Фишером, Хоуксом в классе конечных разрешимых групп был разработан метод экстремальных классов для изучения локальных формаций и их критических групп. В настоящей работе решается задача Л. А. Шеметкова о распространении данного метода на произвольные конечные группы.

Библиография: 6 названий.

Введение. Пусть $\mathfrak{F}$ и $\mathfrak{X}$ - некоторые классы груп. Класс $\mathfrak{F}^{\mathfrak{X}}$ определяется следуюшим образом: группа $G \in \mathfrak{F}^{\mathfrak{X}}$ тогда и только тогда, когда каждая $\mathfrak{X}$-подгрупша из $G$ принадлежит $\mathfrak{F}$. Конструкция класса $\mathfrak{F}^{\mathfrak{X}}$ была введена и изучалась в классе конечных разрешимых груш Картером, Фишером, Хоуксом в [1]. Ими было установлено, что $\mathfrak{F}^{\mathfrak{X}}$ - локальная формация, если $\mathfrak{F}$ - локальная формация и $\mathfrak{X}$ - экстремальный класс. В [1] также доказано, что $\mathfrak{F}^{\mathfrak{X}} \subseteq \mathfrak{F}$, если $\mathfrak{X}$ - экстремальный класс и $\mathfrak{F}$ - локальная формация,имеющая локальный экран $f$ такой, что $f^{\mathfrak{X}}(p) \subseteq f(p)$ для любого простого $p$. Перечисленные выше результаты нашли приложения для изучения минимальных не $\mathfrak{F}$-групп, т.е. групп, у которых данному классу $\mathfrak{F}$ принадлежат только собственные подгрупшы (подробности см. в [2], [3]).

В 1978 году Л. А. Шеметковым была поставлена задача (см. [2, с. 243, проблема 25]): распространить отмеченные вьше утверждения на произвольные (не обязательно разрешимые) локальные формации. В [4] В. Н. Семенчук исследовал эту задачу для $S$-замкнутых локальных формаций. В настоящей работе получено полное решение данной задачи. Установлено, что в общем случае перенос результатов Картера, Фишера, Хоукса на произвольные формации невозможен. В частности, построен пример локальной формации $\mathfrak{F}$ и экстремального класса $\mathfrak{X}$, для которых формация $\mathfrak{F}^{\mathfrak{X}}$ нелокальна. В то же время нами указьваются условия, налагаемые на локальный экран $f$ и группы формации $\mathfrak{F}^{\mathfrak{X}}$, в которых данная задача решается положительно. Существенность предложенных условий подтверждается примерами.

В работе рассматриваются только конечные группы. Напомним [5], что для любой группы $G$ и ее подгруппы Фиттинга $F(G)$ обобщенная подгруппа Фиттинга $F^{*}(G)$ определяется следующим образом: $F^{*}(G) / F(G)=\operatorname{soc}\left(C_{G}(F(G)) F(G) / F(G)\right)$, где sос означает цоколь. Через $E(G)$ обозначается последний член в произвольном ряде группы $F^{*}(G)$. Нам потребуются следуюшие хорошо известные свойства подгрупшы $F^{*}(G)$. Для любой групшы $G$ имеет место $F^{*}(G)=F(G) E(G)$ и $C_{G}\left(F^{*}(G)\right) \subseteq F(G)$ (см. теоремы 13.8 и 13.12 из [5, гл. Х]). 
Необходимые обозначения, определения и результаты из теории формаций можно найти в [2], [3], [6]. Отдельно отметим, что $\mathfrak{F}=\operatorname{LF}(f)$ означает, что $\mathfrak{F}$ - локальная формация, заданная локальным экраном $f$. Локальньй экран $f$ назьвается полньм, если $f(p)=\mathfrak{N}_{p} f(p)$ для любого простого $p$. Пересечение всех (локальных) формаций, содержащих некоторое множество групп $\mathfrak{X}$, называется (соответственно локальной) формацией, порожденной $\mathfrak{X}$. Через form $\mathfrak{X}$ обозначается формация, а через lform $\mathfrak{X}$ - локальная формация, порожденная множеством $\mathfrak{X}$. Везде в работе $\mathfrak{G}$ обозначает класс всех групп, $\mathfrak{S}$ - класс всех разрешимых групп. Если $\mathfrak{X}-$ класс групп и $\pi$ - некоторое множество простых чисел, то $\mathfrak{X}_{\pi}-$ класс всех $\pi$-групп, принадлежаших классу $\mathfrak{X}$. Всюду $\mathscr{P}-$ множество всех простых чисел.

Решение задачи. Центральным моментом работы [1] является понятие экстремального класса. В дальнейшем мы будем использовать предложенное в [2] расширение этого понятия.

ОПРЕДЕЛЕНИЕ 1. Пусть $\mathfrak{H}$ - некоторьй непустой класс групп. Класс групп $\mathfrak{X}$ называется $\mathfrak{H}$-әкстремальным классом, если выполняются следуюшие условия:

1) $\mathfrak{X}$ - непустой насьшенный гомоморф;

2) $\mathfrak{X} \leqslant \mathfrak{H}$;

3) если $\mathfrak{H}$-группа $G$ имеет единственную минимальную нормальную подгруппу $K$ и $G / K \in \mathfrak{X}$, то $G \in \mathfrak{X}$.

Если $\mathfrak{H}=\mathfrak{G}$, то в этом определении $\mathfrak{H}$ опускается, и мы получаем понятие экстремального класса. Понятию экстремального класса, рассматриваемому в [1], будет соответствовать случай, когда $\mathfrak{H}=\mathfrak{S}$.

Лемма 1. Пусть $\mathfrak{F}-$ формация, $\mathfrak{X}-\mathfrak{H}$-әкстремальный класс. Тогда $\mathfrak{F}^{\mathfrak{X}}$ S-замкнутая формачия.

Доказательство следует из определения класса $\mathfrak{F}^{\mathfrak{X}}$ и леммы 25.4 из [2].

Ключевым в данной работе является следующее

ОПРЕДЕЛЕНИЕ 2. Формацию $\mathfrak{F}$ назовем квазинаследственной, если из $G \in \mathfrak{F}$ и $G=H F^{*}(G)$ всегда следует, что $H \in \mathfrak{F}$. Локальный экран $f$ назовем квазинаследственным, если $f(p)$ является квазинаследственной формацией для любого простого $p$.

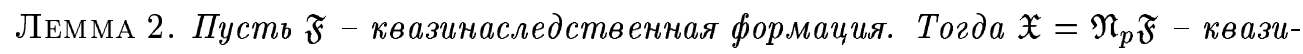
наследственная формачия.

ДокАЗАТЕЛЬСтво. Пусть $G=H F^{*}(G)$ и $G \in \mathfrak{X}$. Тогда

$$
G / O_{p}(G)=H O_{p}(G) / O_{p}(G) \cdot F^{*}(G) / O_{p}(G)=H O_{p}(G) / O_{p}(G) \cdot F^{*}\left(G / O_{p}(G)\right) \in \mathfrak{F}
$$

Из квазинаследственности $\mathfrak{F}$ следует, что $H O_{p}(G) / O_{p}(G) \simeq H / H \cap O_{p}(G) \in \mathfrak{F}$. Но тогда $H \in \mathfrak{N}_{p} \mathfrak{F}=\mathfrak{X}$. Лемма доказана.

В дальнейшем, если $\mathfrak{X}-\mathfrak{H}$-экстремальньй класс и $f-$ локальньй экран, то через $f^{\mathfrak{X}}$ будем обозначать локальную функцию, определяемую равенством $f^{\mathfrak{X}}(p)=(f(p))^{\mathfrak{X}}$ для любого простого $p$.

ЛЕмма 3. Пусть $\mathfrak{H}-$ непустой класс и $\mathfrak{X}-\mathfrak{H}$-әкстремальный класс. Если $\mathfrak{F}=\operatorname{LF}(f)$, mo $\operatorname{LF}\left(f^{\mathfrak{X}}\right) \subseteq \mathfrak{F}^{\mathfrak{X}}$. 
ДокАЗАТЕЛЬСтво. Пусть $G \in \mathrm{LF}\left(f^{\mathfrak{X}}\right)$ и $R$ - произвольная $\mathfrak{X}$-подгруппа группы $G$. По лемме 4.5 из [2] $G / F_{p}(G) \in f^{\mathfrak{X}}(p)$ для любого простого $p \in \pi(G)$. Так как $R F_{p}(G) / F_{p}(G) \in \mathfrak{X}$, то $R F_{p}(G) / F_{p}(G) \in f(p)$ для любого $p \in \pi(R)$. Но тогда $R / R \cap F_{p}(G) \in f(p)$, а значит, $R / F_{p}(R) \in Q f(p)=f(p)$ для любого $p \in \pi(R)$. Отсюда по лемме 4.5 из [2] следует, что $R \in \mathfrak{F}$. Тогда $G \in \mathfrak{F}^{\mathfrak{X}}$, а значит, $\operatorname{LF}\left(f^{\mathfrak{X}}\right) \subseteq \mathfrak{F}^{\mathfrak{X}}$. Лемма доказана.

ТЕОРема 1. Пусть $\mathfrak{H}-$ локальная $S$-замкнутая формачия, $\mathfrak{X}-\mathfrak{H}$-экстремальный класс и $\mathfrak{F}=\mathrm{LF}(f)$, где $f-$ квазинаследственный локальный әкран. Тогда $\mathfrak{F}^{\mathfrak{X}} \cap \mathfrak{H}=$ $\operatorname{LF}\left(\varphi^{\mathfrak{X}}\right) \cap \mathfrak{H}$, где $\varphi$ - такой локальныцй әкран, что $\varphi(p)=\mathfrak{N}_{p} f(p)$ для любого просmoго $p$.

ДокАЗАТЕЛЬСТво. Пусть $\varphi$ - локальньй экран такой, что $\varphi(p)=\mathfrak{N}_{p} f(p)$ для любого простого $p$. Так как $f$-квазинаследственный локальный экран, то по лемме 2 функция $\varphi$ также является квазинаследственным экраном. По лемме 3.13 из [2] $\mathfrak{F}=\mathrm{LF}(f)=$ $\operatorname{LF}(\varphi)$.

Обозначим $\mathfrak{F}^{*}=\operatorname{LF}\left(\varphi^{\mathfrak{X}}\right)$. Из леммы 3 следует, что $\mathfrak{F}^{*} \subseteq \mathfrak{F}^{\mathfrak{X}}$. Тогда $\mathfrak{F}^{*} \cap \mathfrak{H} \subseteq \mathfrak{F}^{\mathfrak{X}} \cap \mathfrak{H}$. Докажем обратное включение $\mathfrak{F}^{\mathfrak{X}} \cap \mathfrak{H} \subseteq \mathfrak{F}^{*} \cap \mathfrak{H}$. Предположим, что множество $\left(\mathfrak{F}^{\mathfrak{X}} \cap \mathfrak{H}\right) \backslash\left(\mathfrak{F}^{*} \cap \mathfrak{H}\right)$ не пусто, и выберем в нем группу $G$ наименьшего порядка. Так как классы $\mathfrak{F}^{\mathfrak{X}} \cap \mathfrak{H}$ и $\mathfrak{F}^{*} \cap \mathfrak{H}$ являются формациями, то $G$ имеет единственную минимальную нормальную подгруппу $N$, совпадающую с $\left(\mathfrak{F}^{*} \cap \mathfrak{H}\right)$ - корадикалом групшы $G$. Так как формация $\mathfrak{F}^{*} \cap \mathfrak{H}$ локальна, то $\Phi(G)=1$. Рассмотрим два случая.

1. Пусть $F(G) \neq 1$. Тогда из $\Phi(G)=1$ и единственности $N$ следует, что $N$ - абелева $p$-группа, где $p$ - некоторое простое число и $F^{*}(G)=F(G)=N$. Пусть $C / N-$ $\mathfrak{X}$-подгрупша грушшы $C / N$. Предположим, что $C=G$. Из $S$-замкнутости формации $\mathfrak{F}^{\mathfrak{X}} \cap \mathfrak{H}$ и выбора $G$ следует, что $C \in \mathfrak{F}^{*} \cap \mathfrak{H}$. Тогда по лемме 4.5 из $[2] C / F_{p}(C) \in \varphi^{\mathfrak{X}}(p)$, где $p \in \pi(N)$. так как $N \subseteq F_{p}(C)$ и $\mathfrak{X}=Q \mathfrak{X}$, то $C / F_{p}(C) \in \mathfrak{X}$. Поэтому $C / F_{p}(C) \in \varphi(p)$. В силу $C_{G}\left(F^{*}(G)\right)=C_{G}(N)=N$ получаем, что $O_{p^{\prime}}(C)=1$. Тогда $F_{p}(C)-p$-группа. Поэтому $C \in \mathfrak{N}_{p} \varphi(p)=\varphi(p)$, а значит, $C / N \in \varphi(p)$. Предположим теперь, что $C / N \in \mathfrak{X}$. Ввиду единственности $N$ и согласно определению $\mathfrak{H}$-экстремального класса, $G \in \mathfrak{X}$. Так как $G \in \mathfrak{F}^{\mathfrak{X}}$, то $G \in \mathfrak{F}$. Но тогда $G / C_{G}(N)=G / N \in \varphi(p)$. Таким образом, мы доказали, что каждая $\mathfrak{X}$-подгруппа из $G / N$ принадлежит $\varphi(p)$. Это означает, что $G / C_{G}(N)=G / N \in \varphi^{\mathfrak{X}}(p)$. Следовательно, $N \varphi^{\mathfrak{X}}$-центральна в $G$. Но тогда $G \in \mathfrak{F}^{*}$, а значит, $G \in \mathfrak{F}^{*} \cap \mathfrak{H}$. Получили противоречие с выбором $G$.

2. Пусть $F(G)=1$. Тогда $F^{*}(G)=\operatorname{soc}(G)$. Из единственности $N$ следует, что $N=F^{*}(G)$. Тогда из $C_{G}\left(F^{*}(G)\right) \subseteq F(G)=1$ имеем, что $C_{G}(N)=1$. Пусть $D-$ произвольная $\mathfrak{X}$-подгруппа из $G$. Рассмотрим подгруппу $R=D N$. Вначале покажем, что $F_{p}(R)=1$ для любого $p \in \pi(N)$. Зафиксируем $p \in \pi(N)$. Заметим, что $O_{p^{\prime}}(R) \subseteq F_{p}(R)$. Так как $N$ - неабелева минимальная нормальная подгруппа из $G$ и $p \in \pi(N)$, то $O_{p^{\prime}}(N)=O_{p}(N)=1$. Поэтому $O_{p^{\prime}}(R) \cap N=O_{p}(R) \cap N=1$. Тогда $\left[O_{p^{\prime}}(R), N\right]=\left[O_{p}(R), N\right]=1$. Отсюда и из $C_{G}(N)=1$ получаем, что $O_{p^{\prime}}(R)=O_{p}(R)=1$. Но тог да $F_{p}(R)=1$ для любого $p \in \pi(N)$. Предположим, что $R \neq G$. В силу выбора $G$ и $S$-замкнутости формации $\mathfrak{F}^{\mathfrak{X}} \cap \mathfrak{H}$ получаем, что $R \in \mathfrak{F}^{*} \cap \mathfrak{H}$. Тогда $R \simeq R / F_{p}(R) \in \varphi^{\mathfrak{X}}(p)$ для любого $p \in \pi(N)$. Отсюда и из $D \in \mathfrak{X}$ следует, что $D \in \varphi(p)$. Пусть теперь $R=G$. Так как $\mathfrak{X}-\mathfrak{H}$-экстремальный класс, то из $G \in \mathfrak{H}$ и $G / N=D N / N \simeq D / D \cap N \in Q \mathfrak{X}=\mathfrak{X}$ получаем, что $G \in \mathfrak{X}$. Следовательно, $G \in \mathfrak{F}$. Тогда $G \simeq G / F_{p}(G) \in \varphi(p)$ для любого $p \in \pi(G)$. Отсюда и из $G=D N=D F^{*}(G)$ ввиду квазинаследственности формации 
$\varphi(p)$ следует, что $D \in \varphi(p)$. Итак, установлено, что всякая $\mathfrak{X}$-подгруппа из $G$ принадлежит формации $\varphi(p)$ для любого $p \in \pi(N)$, т.е. $G \in \varphi^{\mathfrak{X}}(p), p \in \pi(N)$. Из $G / N \in \mathfrak{F}^{*}$ и $G / C_{G}(N) \simeq G \in \varphi^{\mathfrak{X}}(p), p \in \pi(N)$, следует, что $G \in \mathfrak{F}^{*}$. Так как $G \in \mathfrak{H}$, то $G \in \mathfrak{F}^{*} \cap \mathfrak{H}$. Получили противоречие. Теорема доказана.

СлЕДСТВИЕ 1. Пусть $\mathfrak{F}=\mathrm{LF}(f)$, әде $f-$ полный квазинаследственный локальный экран и $\mathfrak{X}$ - әкстремальныц йласс. Тогда $\mathfrak{F}^{\mathfrak{X}}=\operatorname{LF}\left(f^{\mathfrak{X}}\right)$.

Пусть $\mathfrak{F}$ - некоторый класс групा. Если $\mathfrak{F}_{1}$ и $\mathfrak{F}_{2}-S$-замкнутые подклассы из $\mathfrak{F}$, то $\mathfrak{F}_{1} \cup \mathfrak{F}_{2}$ также является $S$-замкнутым подклассом из $\mathfrak{F}$. Поэтому в $\mathfrak{F}$ всегда имеется наибольший (возможно пустой) замкнутый относительно подгрупп подкласс, которьй обозначается через $\mathfrak{F}^{S}$. Заметим, что $\mathfrak{F}^{S}=\mathfrak{F}^{\mathfrak{G}}$. Так как класс всех групп $\mathfrak{G}$, очевидно, является экстремальным, то имеет место следующее

СлЕДСТВИЕ 2. Пусть $\mathfrak{F}=\mathrm{LF}(f)$, где $f-$ полный квазинаследственный локальный әкран. Тогда $\mathfrak{F}^{S}=\operatorname{LF}\left(f^{S}\right)$.

СледствиЕ 3 (Картер, Фишер, Хоукс [1]). Пусть $\mathfrak{F}=\mathrm{LF}(f)$, әде $f-$ полный локальный S-экран. Тогда для любого $\mathfrak{S}$-экстремального класса $\mathfrak{X}$ выполняется $\mathfrak{F}^{\mathfrak{X}} \cap \mathfrak{S}=\operatorname{LF}\left(f^{\mathfrak{X}}\right) \cap \mathfrak{S}$.

ДокАЗАТЕЛЬСтво. Предположим, что $G=H F^{*}(G)$ и $G \in f(p)$, где $p$ - некотоpoе простое число. Так как $f(p) \subseteq \mathfrak{S}$, то из $G \in f(p)$ имеем, что $F^{*}(G)=F(G)$ и $G=H F(G)$. По теореме 2.3 из [2] получаем, что $H \in f(p)$. Следовательно, $f(p)-$ квазинаследственная формация для любого простого $p$. Остается применить теорему 1. Следствие доказано.

ЛЕмма 4. Пусть $\mathfrak{H}-S$-замкнутая формаиия, $\mathfrak{F}-$ квазинаследственная формаиия $и \mathfrak{X}-\mathfrak{H}$-әкстремальны й класс. Если $\mathfrak{F}^{\mathfrak{X}} \cap \mathfrak{H} \subseteq \mathfrak{F} \cap \mathfrak{H}$, mo $\left(\mathfrak{N}_{p} \mathfrak{F}\right)^{\mathfrak{X}} \cap \mathfrak{H} \subseteq \mathfrak{N}_{p} \mathfrak{F} \cap \mathfrak{H}$.

ДокАЗАТЕЛЬСтво. Предположим, что множество $\left(\left(\mathfrak{N}_{p} \mathfrak{F}\right)^{\mathfrak{X}} \cap \mathfrak{H}\right) \backslash\left(\mathfrak{N}_{p} \mathfrak{F} \cap \mathfrak{H}\right)$ не пусто, и выберем в нем группу $G$ наименьшего порядка. Пусть $N$ - минимальная нормальная подгруппа из $G$. Тогда $N$ - единственная минимальная нормальная подгруппа в $G$ и $N=G^{\mathfrak{N}_{p} \mathfrak{F} \cap \mathfrak{H}}$. Пусть $R$ - произвольная $\mathfrak{X}$-подгруппа из $G$. Положим $B=R F^{*}(G)$, и рассмотрим два случая.

1. Пусть $F(G) \neq 1$. Тогда $N$ - абелева $q$-группа, где $q$ - некоторое простое число. Если $q=p$, то из $G / N \in \mathfrak{N}_{p} \mathfrak{F} \cap \mathfrak{H}$ следует, что $G \in \mathfrak{N}_{p} \mathfrak{F} \cap \mathfrak{H}$. Противоречие. Следовательно, $N-q$-группа, где $q \neq p$.

Предположим, что $B \neq G$. Из $S$-замкнутости формации $\left(\mathfrak{N}_{p} \mathfrak{F}\right)^{\mathfrak{X}} \cap \mathfrak{H}$ в силу выбора $G$ следует, что $B \in \mathfrak{N}_{p} \mathfrak{F} \mathfrak{H}$. Так как $N$ - единственная минимальная нормальная подгруппа в $G$ и $\pi(N)=\{q\}$, то $F(G)$ является $q$-группой. Так как $F^{*}(G)=F(G) E(G) \subseteq B$, то $E(G) \subseteq N_{G}\left(O_{p}(B)\right)$. Поэтому по утверждению б) леммы 13.16 из [5] получаем, что $\left[E(G), O_{p}(B)\right]=1$. Очевидно, $\left[F(G), O_{p}(B)\right]=1$. Следовательно, $\left[F^{*}(G), O_{p}(G)\right]=1$, а значит, $O_{p}(B) \subseteq C_{G}\left(F^{*}(G)\right) \subseteq F(G)$. Отсюда следует, что $O_{p}(B)=1$. Но тогда $B \in \mathfrak{F} \cap \mathfrak{H}$. Из $B=R F^{*}(G)=R F^{*}(B)$ и квазинаследственности формации $\mathfrak{F}$ получаем, что $R \in \mathfrak{F}$.

Пусть теперь $B=G$. Если $\Phi(G)=1$, то из единственности $N$ и $N \subseteq F(G)$ следует, что $N=F^{*}(G)=F(G)$. Тогда $G=R N$. Из $G / N=R N / N \simeq R / R \cap N \in Q \mathfrak{X}=\mathfrak{X}$ и определения $\mathfrak{H}$-экстремального класса $\mathfrak{X}$ следует, что $G \in \mathfrak{X}$. Тогда из $G \in\left(\mathfrak{N}_{p} \mathfrak{F}\right)^{\mathfrak{X}}$ получаем, что $G \in \mathfrak{N}_{p} \mathfrak{F}$. Очевидно, что $G \in \mathfrak{N}_{p} \mathfrak{F} \cap \mathfrak{H}$. Получили противоречие. Пусть 
$\Phi(G) \neq 1$. Тогда $N \subseteq \Phi(G)$. Рассмотрим подгруппу $H=R N$. Если $H=G$, то $R=G \in \mathfrak{X}$. Отсюда и из $G \in\left(\mathfrak{N}_{p} \mathfrak{F}\right)^{\mathfrak{X}} \cap \mathfrak{H}$ вытекает, что $G \in \mathfrak{N}_{p} \mathfrak{F} \cap \mathfrak{H}$. Противоречие. Будем считать, что $H \neq G$. Заметим, что $F(G) / N=F(G / N)-q$-группа, $q \neq p$, следовательно, $O_{p}(G / N)=1$. Отсюда и из $G / N \in \mathfrak{N}_{p} \mathfrak{F}$ получаем, что $G / N \in \mathfrak{F}$. Так как $G / N=H F^{*}(G) / N=H / N \cdot F^{*}(G / N) \in \mathfrak{F}$, то из квазинаследственности формации $\mathfrak{F}$ следует, что $H / N \in \mathfrak{F}$. Таким образом, $R / R \cap N \simeq R N / N=H / N \in \mathfrak{F}$. С другой стороны, из $R \in \mathfrak{N}_{p} \mathfrak{F}$ вытекает, что $R / O_{p}(R) \in \mathfrak{F}$. Тогда $R \simeq R / R \cap N \cap O_{p}(R) \in R_{0} \mathfrak{F}=\mathfrak{F}$. Следовательно, мы установили, что любая $\mathfrak{X}$-подгруппа из $G$ принадлежит $\mathfrak{F}$. Тогда $G \in \mathfrak{F}^{\mathfrak{X}}$. Так как $G \in \mathfrak{H}$, то из условия $\mathfrak{F}^{\mathfrak{X}} \cap \mathfrak{H} \subseteq \mathfrak{F} \cap \mathfrak{H}$ следует, что $G \in \mathfrak{F} \cap \mathfrak{H} \subseteq \mathfrak{N}_{p} \mathfrak{F} \cap \mathfrak{H}$. Получили противоречие с выбором $G$.

2. Пусть $F(G)=1$. Тогда $N$ неабелева. Из единственности $N$ следует, что $F^{*}(G)=N$ и $B=R F^{*}(G)=R N$. Ввиду $C_{G}\left(F^{*}(G)\right) \subseteq F(G)$ получаем, что $C_{G}(N)=1$. Если $B=G$, то из $G / N=R N / N \simeq R / R \cap N \in Q \mathfrak{X}=\mathfrak{X}$ следует, что $G \in \mathfrak{X}$. Отсюда и из $G \in\left(\mathfrak{N}_{p} \mathfrak{F}\right)^{\mathfrak{X}} \cap \mathfrak{H}$ получаем, что $G \in \mathfrak{N}_{p} \mathfrak{F} \cap \mathfrak{H}$. Противоречие. Будем считать, что $B \neq G$. Тогда из $S$-замкнутости $\left(\mathfrak{N}_{p} \mathfrak{F}\right)^{\mathfrak{X}} \cap \mathfrak{H}$ и выбора $G$ следует, что $B \in \mathfrak{N}_{p} \mathfrak{F} \cap \mathfrak{H}$. Так как $N \subseteq B$ и $O_{p}(B) \cap N=1$, то $O_{p}(B) \subseteq C_{G}(N)=1$. Поэтому $O_{p}(B)=1$. Следовательно, $B=R N \in \mathfrak{F} \cap \mathfrak{H}$. Так как $N \subseteq F^{*}(B)$, то $B=R F^{*}(B)$. Из квазинаследственности $\mathfrak{F}$ следует, что $R \in \mathfrak{F}$. Тем самым доказано, что любая $\mathfrak{X}$-подгрупша из $G$ принадлежит формации $\mathfrak{F}$. Следовательно, $G \in \mathfrak{F}^{\mathfrak{X}}$. Тогда $G \in \mathfrak{F}^{\mathfrak{X}} \cap \mathfrak{H} \subseteq \mathfrak{F} \cap \mathfrak{H} \subseteq \mathfrak{N}_{p} \mathfrak{F} \cap \mathfrak{H}$. Противоречие. Лемма доказана.

Теорема 2. Пусть $\mathfrak{H}-$ локальная $S$-замкнутая формаиия, $\mathfrak{X}-\mathfrak{H}$-әкстремальный

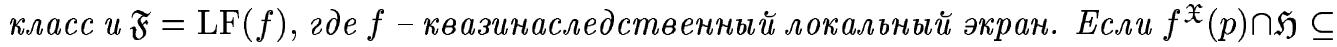
$f(p) \cap \mathfrak{H}$ для любого простого $p$, mо $\mathfrak{F}^{\mathfrak{X}} \cap \mathfrak{H} \subseteq \mathfrak{F} \cap \mathfrak{H}$.

ДокАЗАТЕЛЬСтво. Рассмотрим локальньй экран $\varphi$ такой, что $\varphi(p)=\mathfrak{N}_{p} f(p)$. Тогда по лемме 3.13 из [2] $\mathfrak{F}=\mathrm{LF}(\varphi)$. Ввиду леммы $2 \varphi$-квазинаследственньй локальный экран. Из условия теоремы и леммы 4 вытекает, что $\varphi^{\mathfrak{X}}(p) \cap \mathfrak{H} \subseteq \varphi(p) \cap \mathfrak{H}$. Отсюда и из теоремы 1 следует, что $\mathfrak{F}^{\mathfrak{X}} \cap \mathfrak{H} \subseteq \mathfrak{F} \subseteq \mathfrak{H}$. Теорема доказана.

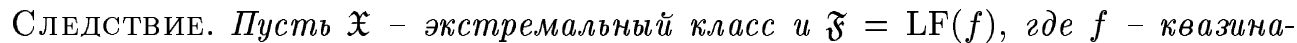
следственный локальный экран. Если $f^{\mathfrak{X}}(p) \subseteq f(p)$ для любого простого $p$, то $\mathfrak{F}^{\mathfrak{X}} \subseteq \mathfrak{F}$.

В случае, когда $\mathfrak{H}=\mathfrak{S}$, из теоремы 2 получаем соответствующий результат из [1].

Дальше мы рассмотрим примеры, показьваюшие, что налагаемые нами требования в теореме 1 являются существенными. Нам потребуется следуюшая

Лемма 5. Пусть $\mathfrak{H}-S$-замкнутая формачия такая, что $\mathfrak{H}=\mathfrak{S} \mathfrak{H}, \mathfrak{X}-\mathfrak{H}$-әкстремальный класс и $\mathfrak{F}=\operatorname{LF}(h)$, где $h$ - максимальный внутренний локальный экран. Если $\mathfrak{F}^{\mathfrak{X}}-$ локальная формачия, то $\mathfrak{F}^{\mathfrak{X}}=\mathrm{LF}\left(h^{\mathfrak{X}}\right)$.

ДокАЗАТЕльСтво. Пусть $\mathfrak{F}^{\mathfrak{X}}$ - локальная формация и $\varphi$ - ее максимальный внутренний локальньй экран. Так как $\mathfrak{F}^{\mathfrak{X}}-S$-замкнутая формация, то по теореме 4.7 из [2] $\varphi-S$-замкнутый экран. По лемме $3 \mathrm{LF}\left(h^{\mathfrak{X}}\right) \subseteq \mathfrak{F}^{\mathfrak{X}}$. Докажем обратное включение. Для этого нам достаточно показать, что $\varphi(p) \subseteq h^{\mathfrak{X}}(p)$ для любого простого $p$. Предположим, что найдется простое число $p$ такое, что множество $\varphi(p) \backslash h^{\mathfrak{X}}(p)$ не пусто. Выберем в нем группу $G$ наименьшего порядка. Так как $\varphi(p)$ и $h^{\mathfrak{X}}(p)$ являются $S$-замкнутьми формациями, то ввиду выбора $G$ является минимальной не $h^{\mathfrak{X}}(p)$-группой. Заметим, что 
$\left(h^{\mathfrak{X}}(p)\right)^{\mathfrak{X}}=h^{\mathfrak{X}}(p)$. Поэтому по лемме 25.5 из [2] $G \in \mathfrak{X}$. Отсюда и из $\varphi(p) \subseteq \mathfrak{F}^{\mathfrak{X}}$ следует, что $G \in \mathfrak{F}$. Отметим также, что в $G$ имеется единственная минимальная нормальная подгруппа. Если $O_{p}(G) \neq 1$, то $G / O_{p}(G) \in h^{\mathfrak{X}}(p)$. Тогда для любой $\mathfrak{X}$-подгруппы $H$ из $G$ вьполняется $H O_{p}(G) / O_{p}(G) \simeq H / H \cap O_{p}(G) \in \mathfrak{X} \cap h(p)$. Так как $h(p)=\mathfrak{N}_{p} h(p)$, то $H \in h(p)$. Следовательно, $G \in h^{\mathfrak{X}}(p)$. Получили противоречие с выбором $G$. Таким образом, $O_{p}(G)=1$. Тогда по лемме 18.8 из [6] существует точный неприводимый $F_{p}[G]$-модуль $L$, где $F_{p}$ - поле из $p$ элементов. Рассмотрим групу $E=L \lambda G$. Из $G \in \mathfrak{X} \subseteq \mathfrak{H}$ и $\mathfrak{H}=\mathfrak{S} \mathfrak{H}$ получаем, что $E \in \mathfrak{H}$. Так как $L$ - единственная минимальная нормальная подгруппа в $E$, то из $E / L \simeq G \in \mathfrak{X}$ и того, что $\mathfrak{X}$ - экстремальньй класс, вытекает, что $E \in \mathfrak{X}$. Кроме того, из $C_{E}(L)=L$ следует, что $E / C_{E}(L)=E / L \simeq G \in \varphi(p) \cap \mathfrak{F}^{\mathfrak{X}}$, а значит, $E \in \mathfrak{F}^{\mathfrak{X}}$. Поэтому $E \in \mathfrak{F}$. Но тог да $E / C_{E}(L) \simeq G \in h(p)$. Отсюда и из $G \in \mathfrak{X}$ в силу выбора $G$ следует, что $G \in h^{\mathfrak{X}}(p)$. Получили противоречие. Лемма доказана.

Следуюший пример показывает, что условие квазинаследственности экрана в теореме 1 отбросить нельзя.

ПримеР 1. Возьмем знакопеременную грушп $A_{5}$ степени 5 и рассмотрим множество $\mathfrak{M}$ всех ее подгрупп. Пусть $\mathfrak{F}=$ lform $\mathfrak{M}$ - локальная формация, порожденная множеством $\mathfrak{M}$. По теореме 8.3 из [6] формация $\mathfrak{F}$ имеет минимальный локальный экран $f$ такой, что $f(p)=\varnothing$, если $p \in \pi^{\prime}(\mathfrak{M})$, и $f(p)=\operatorname{form}\left\{A / F_{p}(A) \mid A \in \mathfrak{M}\right\}$ при всех $p \in \pi(\mathfrak{M})$. Отсюда, учитывая строение групп из $\mathfrak{M}$, находим

$$
f(p)= \begin{cases}\text { form }\left\{C_{3}, A_{5}\right\}, & \text { если } p=2 ; \\ \text { form }\left\{C_{2}, A_{5}\right\}, & \text { если } p=3 ; \\ \text { form }\left\{C_{2}, A_{5}\right\}, & \text { если } p=5 ; \\ \varnothing, & \text { если } p \in \mathscr{P} \backslash\{2,3,5\},\end{cases}
$$

где $C_{p}$ обозначает циклическую группу порядка $p$. Применяя следствие 8.6 из [6], строим максимальньй внутренний локальньй экран $\varphi$ формации $\mathfrak{F}$ такой, что

$$
\varphi(p)= \begin{cases}\mathfrak{N}_{2} \text { form }\left\{C_{3}, A_{5}\right\}, & \text { если } p=2 ; \\ \mathfrak{N}_{3} \text { form }\left\{C_{2}, A_{5}\right\}, & \text { если } p=3 ; \\ \mathfrak{N}_{5} \text { form }\left\{C_{2}, A_{5}\right\}, & \text { если } p=5 ; \\ \varnothing, & \text { если } p \in \mathscr{P} \backslash\{2,3,5\} .\end{cases}
$$

Заметим, что экран $\varphi$ - полный, но не является квазинаследственным. Например, $A_{5}=$ $C_{5} F^{*}\left(A_{5}\right)=C_{5} A_{5} \in \varphi(2)$, но $C_{5} \notin \varphi(2)$.

Для класса всех конечных групп $\mathfrak{G}$, который является экстремальнып, рассмотрим $\mathfrak{F}^{*}=\operatorname{LF}\left(\varphi^{\mathfrak{G}}\right)$. Докажем, что $\mathfrak{F}^{\mathfrak{G}} \neq \mathfrak{F}^{*}$. По лемме $3 \mathfrak{F}^{*} \subseteq \mathfrak{F}^{\mathfrak{G}}$. Поскольку всякая подгрупша из $A_{5}$ принадлежит $\mathfrak{F}$, то $A_{5} \in \mathfrak{F}^{\mathfrak{G}}$. Предположим, что $A_{5} \in \mathfrak{F}^{*}$. Тогда по лемме 4.5 из [2]

$$
A_{5} \simeq A_{5} / F_{2}\left(A_{5}\right) \in \varphi^{\mathfrak{G}}(2)=\left(\mathfrak{N}_{2} \text { form }\left\{C_{3}, A_{5}\right\}\right)^{\mathfrak{G}} .
$$

Это означает, что всякая подгруппа из $A_{5}$ принадлежит $\varphi(2)$. В частности, $C_{5} \in \varphi(2)=$ $\mathfrak{N}_{2}$ form $\left\{C_{3}, A_{5}\right\}$. Тогда $C_{5} \in \operatorname{form}\left\{C_{3}, A_{5}\right\}$, так как $O_{2}\left(C_{5}\right)=1$. Но по теореме 2.2 из [2] form $\left\{C_{3}, A_{5}\right\}=Q R_{0}\left\{C_{3}, A_{5}\right\}$. Откуда нетрудно заметить, что $C_{5} \notin$ form $\left\{C_{3}, A_{5}\right\}$. 
Противоречие. Таким образом, $A_{5} \notin \mathfrak{F}^{*}$. Следовательно, $\mathfrak{F}^{\mathfrak{G}} \neq \mathfrak{F}^{*}$. Отсюда и из леммы 5 получаем, что $\mathfrak{F}^{\mathfrak{G}}$ не является локальной формацией. Так как $\mathfrak{F}^{\mathfrak{G}}=\mathfrak{F}^{S}$, то $\mathfrak{F}-$ пример локальной формации с нелокальной подформацией $\mathfrak{F}^{S}$.

В заключении теоремы 1 утверждается, что $\mathfrak{F}^{\mathfrak{X}} \cap \mathfrak{H}=\operatorname{LF}\left(\varphi^{\mathfrak{X}}\right) \cap \mathfrak{H}$. Так как по условию $\mathfrak{H}$ - локальная формация и пересечение локальных формаций является локальной формацией, то возникает следующий естественньй вопрос: нельзя ли заключение теоремы 1 заменить более сильным утверждением $\mathfrak{F}^{\mathfrak{X}}=\operatorname{LF}\left(\varphi^{\mathfrak{X}}\right)$ ? Отрицательньй ответ на этот вопрос дает следуюший

ПРИмеР 2. Пусть $\mathfrak{H}=\mathfrak{S}$. Ясно, что $\mathfrak{H}$ является $\mathfrak{S}$-экстремальным классом. Зададим локальный экран $f$ следующим образом:

$$
f(p)= \begin{cases}\mathfrak{S}_{\{2,3\}}, & \text { если } p=2 ; \\ \mathfrak{S}_{\{2,3\}}, & \text { если } p=3 ; \\ \mathfrak{S}_{\{2,5\}}, & \text { если } p=5 ; \\ \varnothing, & \text { если } p \in \mathscr{P} \backslash\{2,3,5\} .\end{cases}
$$

Пусть $\mathfrak{F}=\operatorname{LF}(f)$ и $G \in \mathfrak{F}$. Тогда $G / F_{p}(G) \in f(p)$ для любого $p \in \pi(G)$. Из $f(p) \subseteq \mathfrak{S}$ следует, что $G / F_{p}(G)$ - разрешимая группа для любого простого $p \in \pi(G)$. Так как $F(G)=\cap F_{p}(G)$, где $p \in \pi(G)$, то $G / F(G) \in \mathfrak{S}$, а значит, $G$ разрешима. Следовательно, $\mathfrak{F} \subseteq \mathfrak{S}$. Пусть $h$ - максимальньй внутренний локальный экран формации $\mathfrak{F}$. Нетрудно показать, что

$$
h(p)= \begin{cases}\mathfrak{S}_{\{2,3\}}, & \text { если } p=2 ; \\ \mathfrak{S}_{\{2,3\}}, & \text { если } p=3 ; \\ \mathfrak{N}_{5}\left(\mathfrak{F} \cap \mathfrak{S}_{\{2,5\}}\right), & \text { если } p=5 ; \\ \varnothing, & \text { если } p \in \mathscr{P} \backslash\{2,3,5\} .\end{cases}
$$

Рассмотрим $\mathrm{LF}\left(h^{\mathfrak{S}}\right)$. Используя теорему Бернсайда о разрешимости бипримарных групп, нетрудно видеть, что $h^{\mathfrak{S}}(p)=h(p)$ для любого простого $p$. Следовательно, $\mathrm{LF}\left(h^{\mathfrak{S}}\right)=\mathfrak{F} \subseteq \mathfrak{S}$. $\mathrm{C}$ другой стороны, заметим, что $A_{5} \in \mathfrak{F}^{\mathfrak{S}}$. Следовательно, $\mathfrak{F}^{\mathfrak{S}} \neq \mathrm{LF}\left(h^{\mathfrak{S}}\right)$. Отсюда и из леммы 5 получаем, что $\mathfrak{F}^{\mathfrak{S}}$ нелокальна.

\section{СПИСОК ЦИТИРОВАННОЙ ЛИТЕРАТУРЫ}

[1] Carter R., FischerB., Hawkes T. Extreme classes of finite soluble groups // J. Algebra. 1968. V. 9. № 3. P. 285-313.

[2] Шеметков Л. А. Формации конечных групп. М.: Наука, 1978.

[3] Doerk K., Hawkes T. Finite Soluble Groups. Berlin-New York: Walter de Gruyter, 1972.

[4] Семенчук В.Н. Минимальные не $\mathfrak{F}$-группы // Алгебра и логика. 1979. Т. 18.№ 3. C. $348-382$.

[5] Huppert B., Blackburn N. Finite Groups. III. Berlin-Heidelberg-New York: Springer, 1982.

[6] Шеметков Л. А., Скиба А. Н. Формации алгебраических систем. М.: Наука, 1990. 